Supporting Information

\title{
Gold Nanoparticle Quantitation by Whole Cell
}

\section{Tomography}

Aric W. Sanders ${ }^{\ddagger 1, *}$, Kavita M. Jeerage $e^{\ddagger, 2}$, Cindi L. Schwartz ${ }^{t, 3}$, Alexandra E. Curtin ${ }^{1,2}$, Ann N. Chiaramonti $^{2}$

${ }^{1}$ Quantum Electronics and Photonics Division, National Institute of Standards and Technology (NIST), Boulder Colorado, USA

${ }^{2}$ Applied Chemicals and Materials Division, National Institute of Standards and Technology (NIST), Boulder, Colorado, USA

${ }^{3}$ Department of Molecular, Cell, and Developmental Biology, University of Colorado, Boulder, Colorado, USA

*Address correspondence to $\underline{\text { aric.sanders@ } @ \text { nist.gov }}$

${ }^{\ddagger}$ Authors contributed equally to this work

${ }^{1}$ Author now at Electron Microscopy Unit, Rocky Mountain Laboratories, National Institute of Allergy and Infectious Diseases, National Institutes of Health, Hamilton, Montana, USA 
Nanoparticle Dispersion and Characterization of Suspensions. Citrate-stabilized, zerovalent, spherical GNP reference materials (https://www-s.nist.gov/srmors/reports/8013.pdf) had a nominal diameter of $60 \mathrm{~nm}$. Their diameter was characterized by multiple techniques in dry and suspended forms (6 techniques in all), and the average of these values, $55.4 \mathrm{~nm}$, was employed for all calculations. GNPs were dispersed by adding $2.5 \mathrm{~mL}$ of reference material $(51.86 \mu \mathrm{g} / \mathrm{mL})$ to a $50 \mathrm{~mL}$ centrifuge tube containing $2.5 \mathrm{~mL}$ of differentiation medium while vortexing at 1900 rpm. Vortexing continued for $30 \mathrm{~s}$ to surround the GNPs with bovine serum albumin. While many cells are cultured in medium containing $10 \%$ serum, differentiation medium for neural stem cells contains $1 \%$ serum, which provides approximately $0.2 \mathrm{mg} / \mathrm{mL}$ bovine serum albumin.

For dynamic light scattering (DLS), $45 \mu \mathrm{L}$ aliquots were added to a low-volume quartz cuvette (ZEN2112) for measurements with a Zetasizer Nano (Malvern). Backscatter measurements were performed at $20{ }^{\circ} \mathrm{C}$. Stocks were diluted with deionized water or differentiation medium to obtain $5.2 \mu \mathrm{g} / \mathrm{mL}$ suspensions for size analysis. Intensity size distributions (Figure S1A) indicated that the $\mathrm{z}$-average diameter in deionized water, $55.7 \mathrm{~nm}$, was within the reported uncertainty of the reference value for this technique. Once dispersed into low-serum culture medium, the intensity size distribution shifted. The z-average diameter increased to $97.2 \mathrm{~nm}$ and the polydispersity index increased from 0.14 to 0.28 . Differentiation medium without GNPs generated a peak at approximately $10 \mathrm{~nm}$ due to serum proteins. Our results indicate the adsorption of serum proteins and minimal agglomeration.

GNPs in differentiation medium $(5.2 \mu \mathrm{g} / \mathrm{mL} \mathrm{Au})$ were also drop cast onto an ultrathin (less than $3 \mathrm{~nm}$ ) carbon membrane on a holey carbon coated copper support grid (Pelco 01824) for TEM. To prepare each grid, a $15 \mu \mathrm{L}$ aliquot was added to the copper-colored side of the grid and allowed to dry overnight in a biological safety cabinet. Excess fluid was wicked after 30 min to 
minimize the formation of salt crystals which obscure particles in the resulting images. TEM images obtained with a $200 \mathrm{kV}$ Schottky field emission instrument demonstrate that primary particles can be easily identified within particle clusters (Figure S1B).
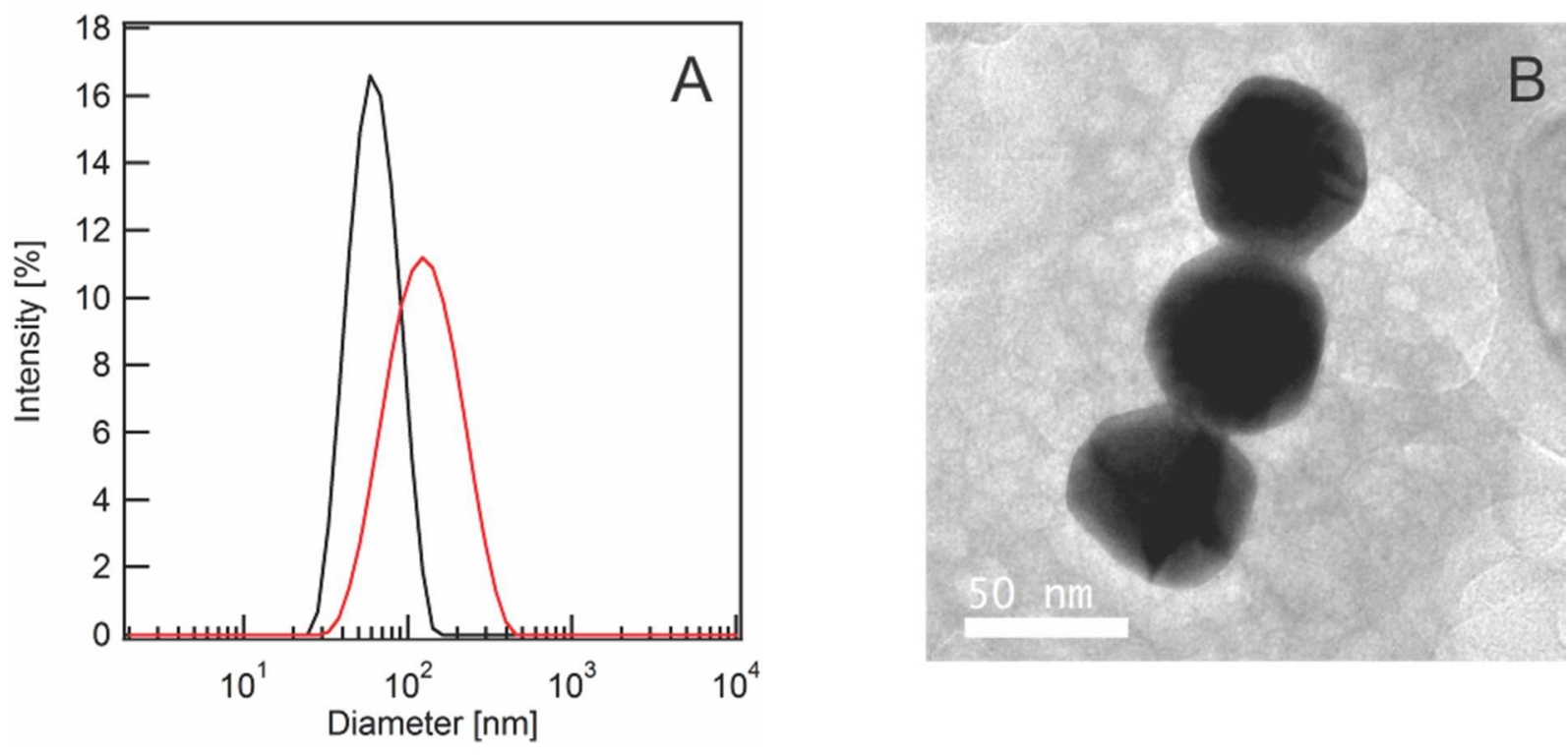

Figure S1. DLS intensity size distributions of $60 \mathrm{~nm}$ GNPs in water (black curve) and in low-serum differentiation medium containing serum proteins (red curve). Distributions were obtained within thirty minutes of dispersion (A). TEM image of $60 \mathrm{~nm}$ gold nanoparticles dropcast from low-serum differentiation medium (B).

Particle Tracking and Quantitation of Nanoparticle Clusters. All analysis began by examining the entire tomographic series in ImageJ to identify sections containing GNPs. Two clusters will be discussed in more detail here: the small cluster identified in Cell 2 (Figure 3) and located in sections 715 - 727 and the large cluster identified in Cell 3 (Figure 5) and located in sections $67-75$. These sections were scaled to increase contrast and viewed in either ImageJ or Neurolucida (MBF Bioscience). To identify unique GNPs imaged by whole cell tomography, we first verified that each successive section images further into the cell interior by measuring the 
distance from a prominent exterior feature to the cell edge (Figure S2A). We then overlaid "finesectioned" images in sequence, for example 715-716-717 and 717-718-719, and marked the nanoparticles in each individual image. By this process we tracked individual particles across multiple sections (Figure S2B). To quantify unique GNPs imaged by whole cell tomography, we needed to verify that each nanoparticle was only present in one section. This was challenging for large clusters due to particle density. Therefore, choosing sections with a small cluster, we overlaid "coarse-sectioned" images in sequence and marked the nanoparticles in each individual image. We found that shine through could contribute to double counting particles. Therefore, only well-defined nanoparticles were marked and counted. Here, we present an independent evaluation of one large cluster (Figure S3).
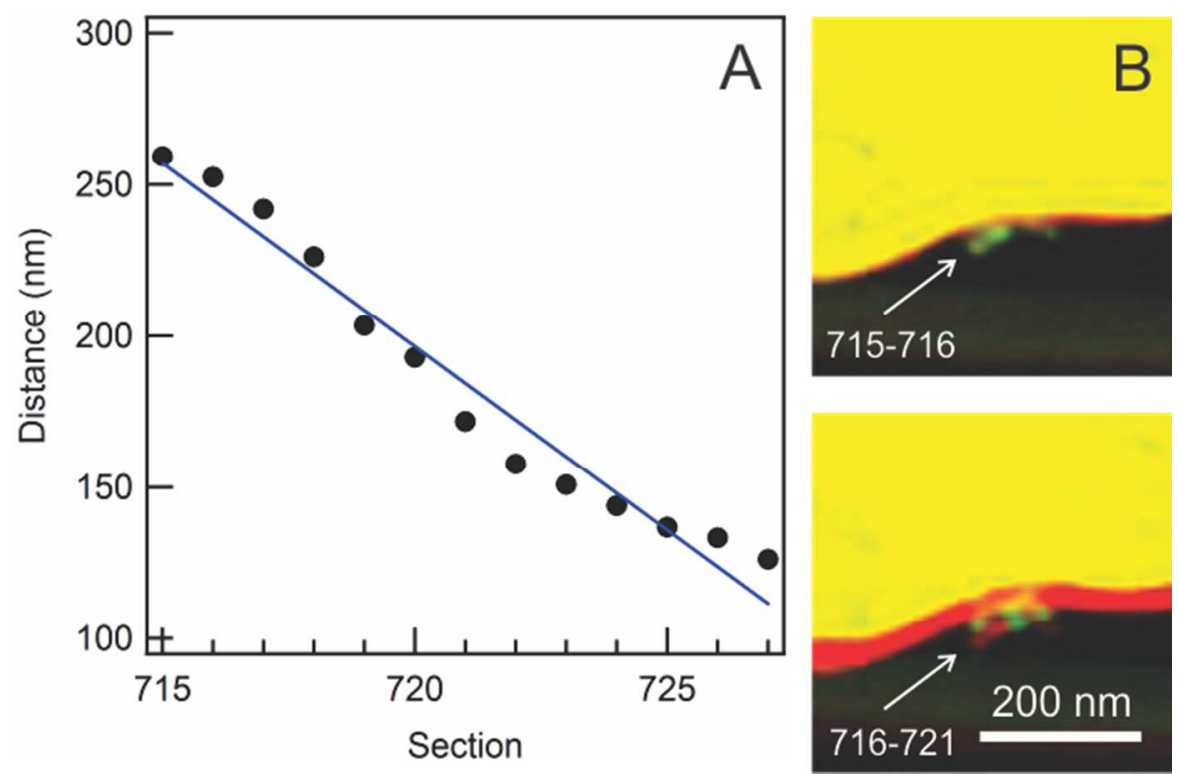

Figure S2. Distance between an exterior feature and the cell edge decreases with each successive section, as expected (A). Highly magnified overlays can be used to track particles (B). In the upper image, the same two particles appear in images 715 (red) and 716 (green). In the lower image, the two particles in image 716 (red) are not found in image 721 (green). Instead several new particles are seen. 

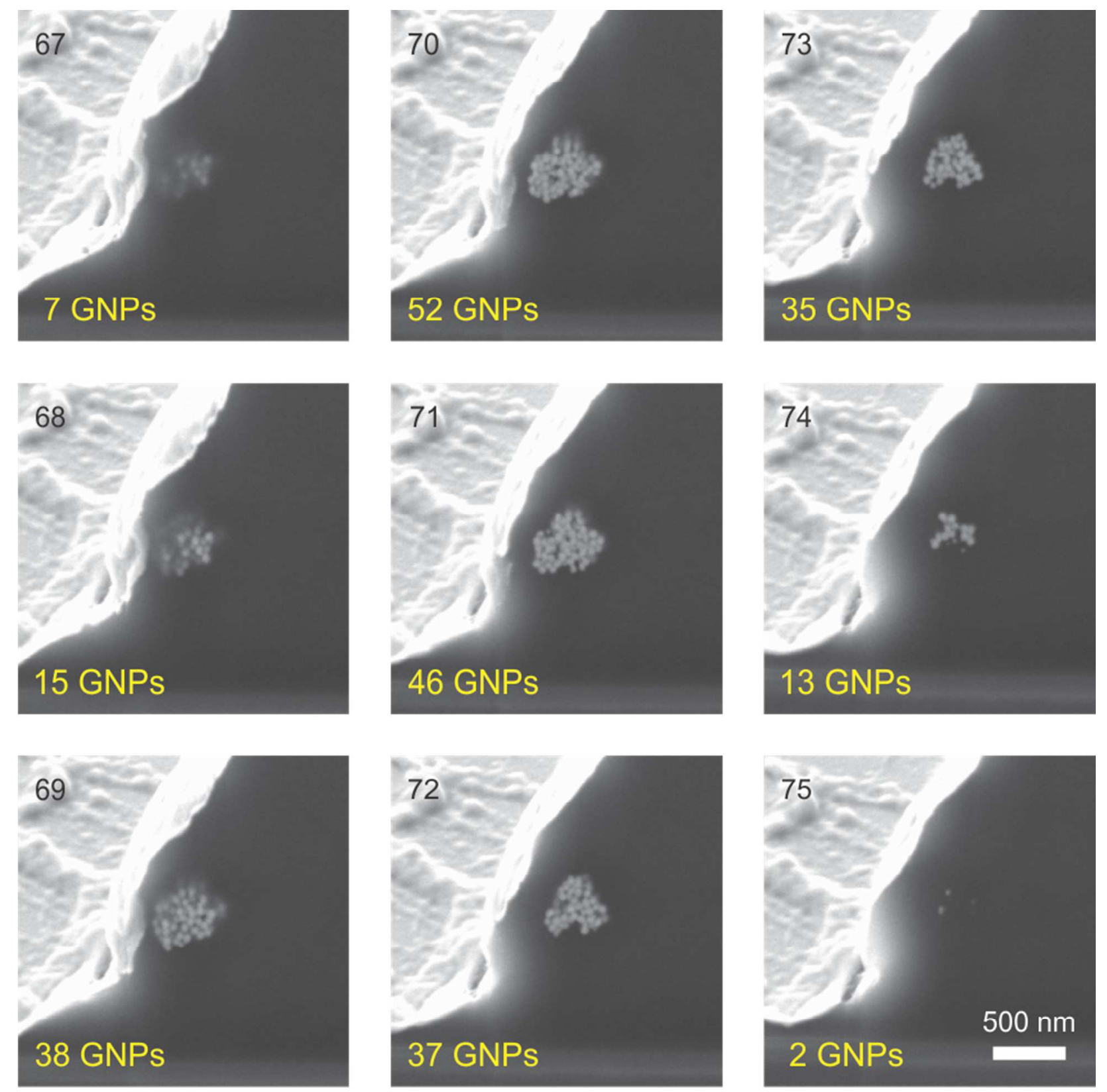

Figure S3. SEM images of a cluster from Cell 3 containing $244 \pm 10$ GNPs. All images from this cluster are shown, with the section indicated in the upper left corner. These images show a portion of the cell membrane (cell exterior, light gray) in the upper left corner, the cell interior (black), and the substrate (dark gray). GNPs were labeled in each section repeatedly and average counts are reported. Shine through from GNPs counted in later sections is apparent, 
particularly in image 68. All images were scaled to increase contrast and identically cropped around the GNP cluster.

Imaging Nanoparticle Clusters with Cellular Contrast. A replicate in vitro sample was prepared following the protocol already described for TEM samples, but stopping after the cells were embedded in plastic and the coverslip glass was dissolved (i.e., individual cells were not excised and thin sectioned). The plastic blocks were mounted and sputter coated following the protocol already described for FIB tomography. These samples were sectioned and imaged with minor modification to the protocols developed for non-embedded cells (Figure S4).
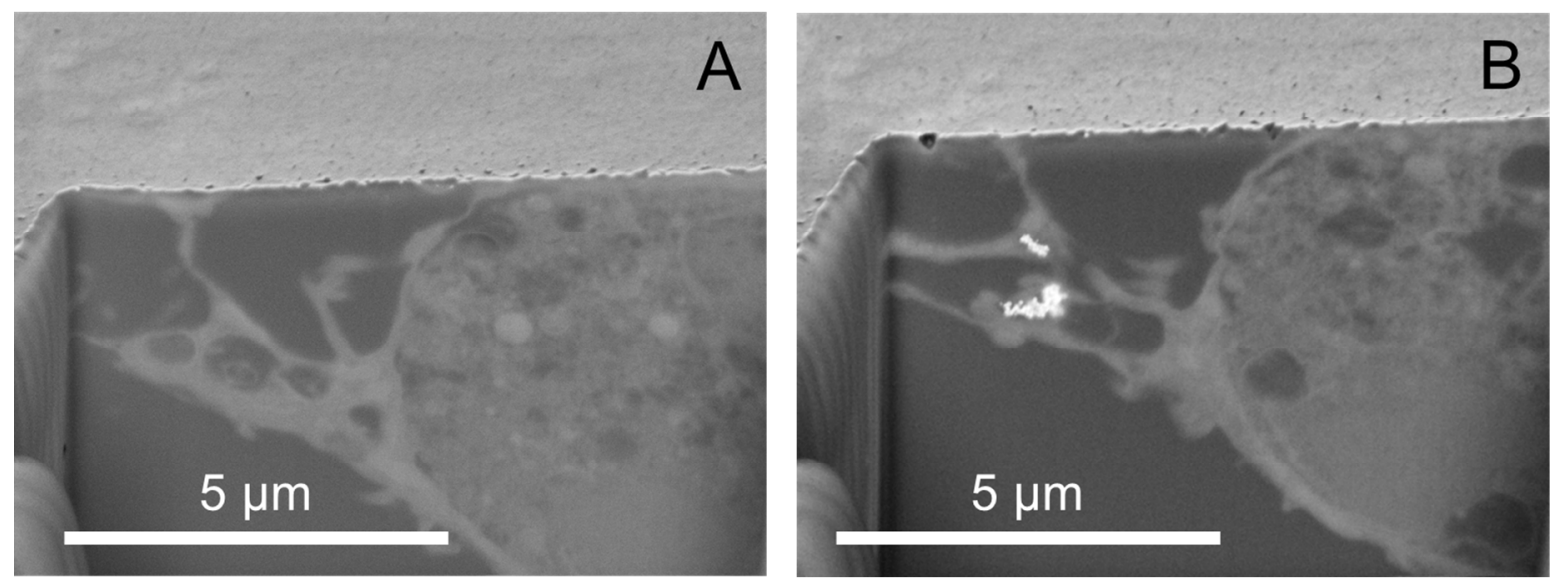

Figure S4. SEM images of FIB cross sections of stained, embedded cells. The cross section in (A) demonstrates the relative contrast of the internal cell membranes and the embedding media. The cluster of nanoparticles in (B) was found $1.44 \mu \mathrm{m}$ further into the sample. Both images were acquired using the same current and acceleration voltage as the images in Figure 4, but the acquisition time was increased to $60 \mathrm{~s}$ to improve signal-to-noise. 